\title{
Flipped classroom - what feedback in the FFL classroom and how to assess the work of learners?
}

\section{[Les classes inversées : quelle rétroaction en salle de FLE et comment évaluer les travaux des apprenant.e.s ?]}

\author{
Cynthia Eid
}

DOI: $10.18355 /$ XL.2021.14.04.02

\begin{abstract}
Assessment is not an additional act in the ecology of the classroom. Evaluative gestures, inseparable from those of education or training, encourage the practitioner to reflect on the scope of their actions and to consider the type of interaction that they promote in the classroom.

If the flipped classroom uses summative assessment, the fact remains that the sinew of war is a formative assessment in the pedagogy of support, encouragement, and benevolence.

We will see in this article what is the act of evaluating, the difference between evaluation of learning and evaluation of teaching by learners, and the different evaluation functions in a flipped classroom.
\end{abstract}

Key words: evaluation functions, the act of evaluating, support, humanistic evaluation, formative evaluation

\section{Résumé}

L'évaluation n'est pas un acte supplémentaire dans l'écologie de la classe. Les gestes évaluatifs, indissociables de ceux de l'éducation ou de la formation, incitent le praticien à réfléchir sur la portée de ses actes et à envisager le type d'interaction qu'il favorise en classe.

Si la classe inversée a recours à l'évaluation sommative, il n'en demeure pas moins que le nerf de la guerre est l'évaluation formative/formatrice dans une pédagogie de l'accompagnement, de l'encouragement et de la bienveillance.

Nous verrons dans cet article ce qu'est l'acte d'évaluer, la différence entre évaluation des apprentissages et évaluation des enseignements par les apprenant.e.s et les différentes fonctions d'évaluation dans une classe inversée.

Mots-clés : fonctions d'évaluation, acte d'évaluer, accompagnement, évaluation humaniste, évaluation formative

\section{Introduction}

En parlant d'alignement pédagogique ${ }^{1}$, quatre éléments du design déterminent la cohérence $^{2}$ d'une activité pédagogique à savoir 1) les objectifs attendus ou les compétences visées, 2) la méthode pédagogique, 3) l'outil ou les outils utilisé(s) et 4) l'évaluation.

\footnotetext{
${ }^{1}$ Selon Biggs Un bon système pédagogique cherche à lier les activités d'enseignementapprentissage et les activités d'évaluation aux objectifs de sorte que tous les aspects du système s'accordent à appuyer l'apprentissage des apprenants (Traduction libre - Biggs, 1999: 11). Biggs est professeur de psychopédagogie en Australie, en Grande Bretagne et à Hong Kong.

${ }^{2}$ La cohérence suppose qu'il y a toujours un rapport étroit entre ce qui est évalué et ce qui a fait l'objet d'apprentissage.
}

XLinguae, Volume 14 Issue 4, October 2021, ISSN 1337-8384, eISSN 2453-711 \5 


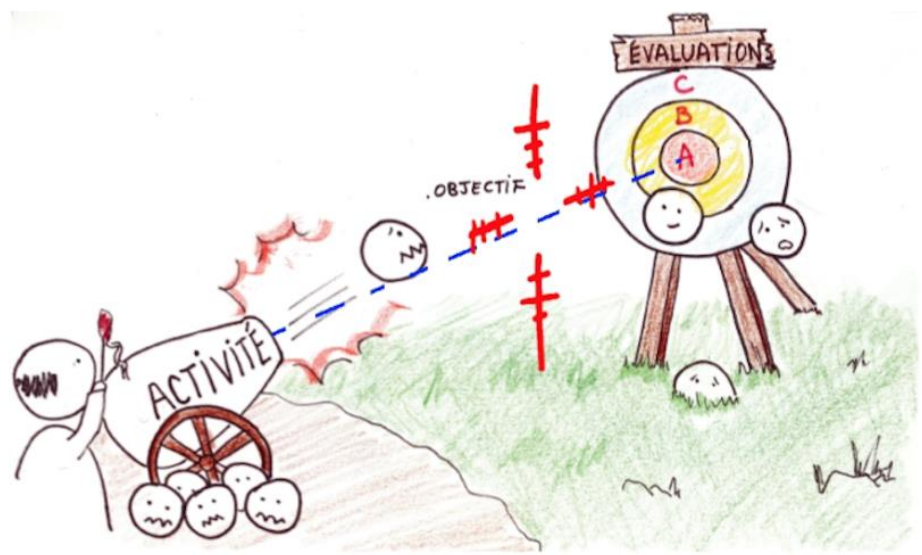

Figure 1. Alignement pédagogique, Tuyet Tram DANG GNOC

Nous ne pouvons pas aborder la classe inversée sans évoquer la question centrale de l'évaluation et sa diversité, et plus spécifiquement la rétroaction et la remédiation, car si a priori la classe est inversée, c'est pour autant inverser l'échec !

Or, en pensant d'une façon générale à l'évaluation, nous avons souvent tendance à privilégier l'évaluation sommative ou certificative (qui nécessite une note) à l'évaluation formative ou formatrice qui met en valeur la pédagogie de l'accompagnement (qui ne nécessite pas de note). Quelques fois la rétroaction ou la remédiation passent inaperçues!

Si la classe inversée a recours à l'évaluation sommative, il n'en demeure pas moins que le nerf de la guerre est l'évaluation formative/formatrice dans une pédagogie de l'accompagnement.

\section{Qu'est-ce que l'acte d'évaluer dans une classe inversée ?}

L'évaluation en général constitue l'un des aspects les plus délicats et les plus difficiles du métier d'enseignant.e. L'évaluation au sein des pédagogies actives (et plus particulièrement au sein d'une classe inversée) diffère-t-elle réellement d'une évaluation classique?

La réponse est nuancée. L'évaluation au sein d'une classe inversée mise davantage, sans négliger l'évaluation sommative, sur la pédagogie de l'accompagnement et de l'encouragement de l'apprenant.e tout le long de son processus d'apprentissage, ce qui facilite grandement la démarche d'apprentissage et aboutit à des évaluations sommatives en général plus performantes.

Avant de rentrer dans les détails de cette question, il serait pertinent d'examiner la définition de l'acte d'évaluer dans une classe inversée.

Dans le tableau ci-dessous ${ }^{3}$ se trouve la définition de l'évaluation (quoi ?), les raisons pour lesquelles elle est utilisée (pourquoi ?), le déroulement des tâches (comment?), à quel moment se pratique-t-elle (quand ?) ainsi que des exemples d'activités utilisées en évaluation (activités ?).

\footnotetext{
${ }^{3}$ Inspiré de Musial, M., Pradère, F., Tricot, A. 2012. Comment concevoir un enseignement ? Bruxelles, De Boeck.
} 


\begin{tabular}{|c|c|c|c|c|}
\hline Quoi ? & Pourquoi? & Comment? & Quand? & Activités? \\
\hline $\begin{array}{l}\text { Situer un } \\
\text { acte (activité, } \\
\text { une } \\
\text { performance, } \\
\text { une } \\
\text { production } \\
\text { d'un } \\
\text { apprenant, } \\
\text { etc.) par } \\
\text { rapport à } \\
\text { une référence } \\
\text { (le progrès de } \\
\text { l'apprenant, } \\
\text { son } \\
\text { classement } \\
\text { par rapport à } \\
\text { la moyenne de } \\
\text { son âge). }\end{array}$ & $\begin{array}{l}\text { Motiver } \\
\text { (l'évaluation } \\
\text { est utilisée } \\
\text { comme moteur } \\
\text { de } \\
\text { l'apprentissage } \\
\text {, focaliser } \\
\text { l'évaluation } \\
\text { sur les progrès } \\
\text { réalisés par } \\
\text { l'apprenant). } \\
\text { Valider } \\
\text { (évaluer pour } \\
\text { vérifier que le } \\
\text { but visé a été } \\
\text { atteint). } \\
\text { Prendre } \\
\text { conscience } \\
\text { (réguler son } \\
\text { propre } \\
\text { apprentissage) }\end{array}$ & $\begin{array}{l}\text { De façon non } \\
\text { intrusive } \\
\text { (évaluer les } \\
\text { apprentissage } \\
\text { s pendant } \\
\text { l'acte } \\
\text { d'apprentissa } \\
\text { ge, pendant } \\
\text { que les } \\
\text { apprenants } \\
\text { travaillent). }\end{array}$ & $\begin{array}{l}\text { En début } \\
\text { d'apprentissa } \\
\text { ge } \\
\text { (l'évaluation } \\
\text { est plutôt } \\
\text { diagnostique, } \\
\text { elle porte sur } \\
\text { l'état des } \\
\text { connaissances } \\
\text { avant que } \\
\text { l'enseignemen } \\
\text { t ne } \\
\text { commence). }\end{array}$ & $\begin{array}{l}\text { Les activités } \\
\text { peuvent être } \\
\text { conçues } \\
\text { pour } \\
\text { diagnostiqu } \\
\text { er les acquis } \\
\text { et les } \\
\text { compétence } \\
\text { s des } \\
\text { apprenants }{ }^{4} \\
\text { (QCM, } \\
\text { Quiz, } \\
\text { exercices } \\
\text { d'apparieme } \\
\text { nt, etc.) }\end{array}$ \\
\hline $\begin{array}{l}\text { Juger de la } \\
\text { différence } \\
\text { entre l'acte et } \\
\text { la référence } \\
\text { (c'est une } \\
\text { mesure). }\end{array}$ & $\begin{array}{l}\text { Aider à } \\
\text { apprendre } \\
\text { (évaluer pour } \\
\text { fournir à } \\
\text { l'apprenant } \\
\text { une aide } \\
\text { précise et } \\
\text { personnalisée } \\
\text { à son } \\
\text { apprentissage) }\end{array}$ & $\begin{array}{l}\text { De façon } \\
\text { intrusive } \\
\text { (évaluer les } \\
\text { apprentissage } \\
\text { s consiste à } \\
\text { prescrire des } \\
\text { tâches } \\
\text { spécifiques } \\
\text { pour } \\
\text { l'évaluation) }\end{array}$ & $\begin{array}{l}\text { En cours } \\
\text { d'apprentissa } \\
\text { ge } \\
\text { (l'évaluation } \\
\text { permet de } \\
\text { réguler } \\
\text { l'apprentissag } \\
\text { e, que ce soit } \\
\text { pour aider } \\
\text { l'apprenant à } \\
\text { prendre } \\
\text { conscience de } \\
\text { ce qu'il a déjà̀ } \\
\text { fait (appris, } \\
\text { produit, } \\
\text { réalisé) ou de } \\
\text { ce qui lui reste } \\
\text { à faire (à } \\
\text { apprendre). }\end{array}$ & $\begin{array}{l}\text { Les activités } \\
\text { de } \\
\text { l'évaluation } \\
\text { formative/ } \\
\text { formatrice } \\
\text { peuvent être } \\
\text { conçues } \\
\text { pour } \\
\text { accompagn } \\
\text { er et } \\
\text { encourager } \\
\text { les } \\
\text { apprenants } \\
\text { dans leurs } \\
\text { démarches } \\
\text { d'apprentiss } \\
\text { age, de } \\
\text { développer } \\
\text { leur plein } \\
\text { potentiel } \\
\text { (régulation } \\
\text { immédiate, } \\
\text { conseils, } \\
\text { etc.) }\end{array}$ \\
\hline
\end{tabular}

${ }^{4}$ Confère infra l'évaluation diagnostique.

${ }^{5}$ Confère infra l'évaluation formative/formatrice.

XLinguae, Volume 14 Issue 4, October 2021, ISSN 1337-8384, eISSN 2453-711 7 


\begin{tabular}{|c|c|c|c|c|}
\hline $\begin{array}{l}\text { L'acte est un } \\
\text { indice d'une } \\
\text { connaissance } \\
\text { ou d'une } \\
\text { compétence. } \\
\text { Partir de ce } \\
\text { qui est } \\
\text { observable } \\
\text { (l'acte, la } \\
\text { performance, } \\
\text { le } \\
\text { comportement } \\
\text { ) et inférer la } \\
\text { connaissance } \\
\text { ou la } \\
\text { compétence. }\end{array}$ & $\begin{array}{l}\text { Dépister ou } \\
\text { repérer (dans } \\
\text { le domaine des } \\
\text { troubles du } \\
\text { langage et de } \\
\text { l'apprentissage } \\
\text { ). } \\
\text { Diagnostique } \\
\text { r (établir la } \\
\text { cause d'un } \\
\text { problème, } \\
\text { d'un } \\
\text { dysfonctionne } \\
\text { ment). } \\
\text { Évaluer le } \\
\text { système } \\
\text { (l'établisseme } \\
\text { nt, le dispositif } \\
\text { d'innovation } \\
\text { par exemple, } \\
\text { PISA, etc.) }\end{array}$ & $\begin{array}{l}\text { De façon } \\
\text { standardisée. } \\
\text { (Utiliser des } \\
\text { outils } \\
\text { d'évaluation } \\
\text { non conçus } \\
\text { par } \\
\text { l'enseignant, } \\
\text { par exemple, } \\
\text { faire passer } \\
\text { un « examen } \\
\text { blanc » aux } \\
\text { apprenants »). }\end{array}$ & $\begin{array}{l}\text { En fin } \\
\text { d'apprentissa } \\
\text { ge (validation } \\
\text { de } \\
\text { l'apprentissag } \\
\text { e, un moyen } \\
\text { d'évaluer les } \\
\text { progrès). }\end{array}$ & $\begin{array}{l}\text { Les activités } \\
\text { de } \\
\text { l'évaluation } \\
\text { sommative } \\
\text { peuvent être } \\
\text { conçues } \\
\text { pour donner } \\
\text { une note } \\
\text { finale aux } \\
\text { apprenants. }\end{array}$ \\
\hline
\end{tabular}

Tableau 1. Qu'est-ce que l'acte d'évaluer ?, inspiré de Musial, M., Pradère, F., \& Tricot, A. (2012). Comment concevoir un enseignement?

Ainsi donc, une évaluation est un pont dynamique entre l'apprentissage et l'enseignement (Allal, 2010).

C'est une opération qui consiste à recueillir des données et à poser un jugement sur une personne, un objet, un processus, une situation ou une organisation en comparant les caractéristiques observables à des normes établies, à partir de critères explicites en vue de fournir des informations utiles à la prise de décision dans la poursuite d'un but ou d'un objectif ${ }^{6}$.

\footnotetext{
${ }^{6}$ Inspirée du Rapport du Groupe de suivi sur l'évaluation de l'enseignement, Université de Montréal, (2001).
} 


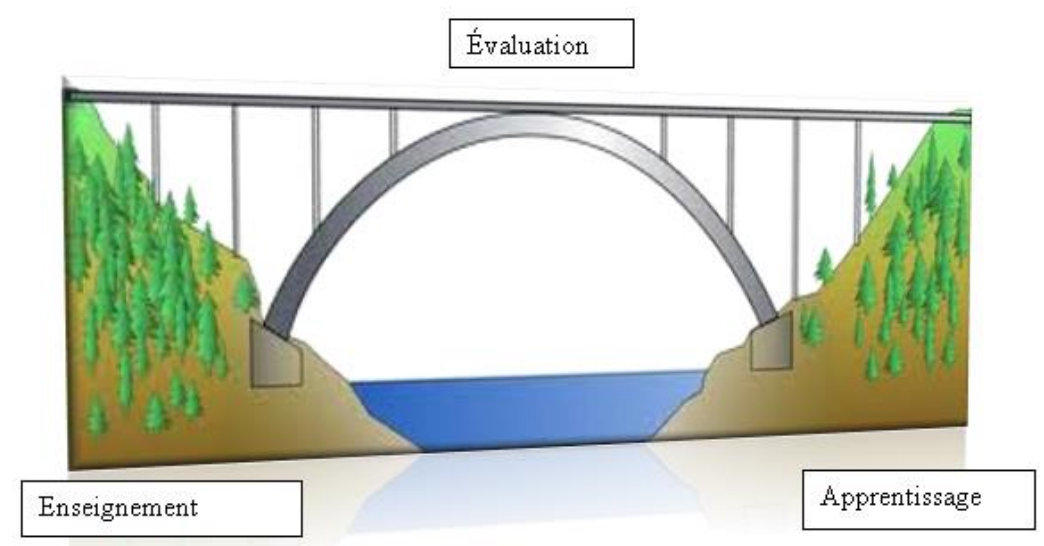

Dans une classe inversée, l'évaluation met en lumière la motivation des apprenant.e.s car elle focalise sur les progrès réalisés, la prise de conscience pour réguler ou autoréguler son apprentissage et l'aide à apprendre à apprendre, une aide précise et personnalisée à son apprentissage. C'est donc la philosophie de l'acte d'évaluer qui diffère dans une classe inversée par rapport à l'acte d'évaluer dans un enseignement frontal.

\section{Quelle est la différence entre évaluation des apprentissages et évaluation des enseignements par les apprenant.e.s ?}

2.1. Qu'est-ce que l'évaluation des apprentissages dans une classe inversée?

La fonction de l'évaluation des apprentissages vise surtout à soutenir l'apprenant.e dans le développement de ses compétences et l'acquisition de connaissances.

«Pour remplir cette fonction, l'évaluation doit être intégrée au processus d'enseignement et jouer un rôle de régulation, c'est-à-dire l'ajustement de l'apprentissage par l'apprenant.e ainsi que l'ajustement des actions pédagogiques par l'enseignant. Les régulations peuvent porter sur différents objets (tests, quiz, exercice, projet, tâche, mémoire, thèse, examens, etc.) et être effectuées à différents moments ${ }^{7} »$. La régulation fera l'objet d'une partie qui sera traitée ci-dessous.

2.2. Qu'est-ce que l'évaluation des enseignements par les apprenant.e.s dans une classe inversée?

L'évaluation des enseignements connaît deux formes :

\footnotetext{
${ }^{7}$ Guide de soutien en évaluation des apprentissages, le ministère de l'Éducation, de l'Enseignement supérieur et de la Recherche, 2015.
}

XLinguae, Volume 14 Issue 4, October 2021, ISSN 1337-8384, eISSN 2453-711 9 
L'évaluation de mi-parcours qui sera traitée dans la partie qui suit et l'évaluation de la prestation d'enseignement par les apprenant.e.s. Ce dernier est un processus formel de collecte des appréciations des apprenants via un questionnaire papier ou un questionnaire en ligne, administré par l'Université en fin de trimestre, d'activité ou de parcours. Elle est surtout utilisée dans l'enseignement supérieur sans exclure d'autres contextes.

Son principal bénéfice est qu'elle peut améliorer la pédagogie des enseignant.e.s et leur renvoyer un miroir réflexif sur leur prestation dans un objectif de régulation (fondement même de la classe inversée).

Pour mieux comprendre l'évaluation des enseignements par les apprenant.e.s, se trouve ci-dessous un exemple d'évaluation d'un cours, à l'Université de Lausanne, consulté en ligne ${ }^{8}$, qui retrace les questions de base posées aux apprenants.

\footnotetext{
${ }^{8}$ [https://www.unil.ch/fbm-up/files/live/sites/fbmup/files/shared/MOD_EVAL_COURS_VI.pdf]
} 


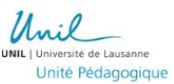

Evaluation du cours :
Donné par :
Dans le cadre du module : $\quad$ Date :

Les résultats de cette évaluation permettront à l'enseignant.e / aux enseignant.e.s de connaître la façon dont vous évaluez cet enseignement et de trouver, le cas échéant, des possibilités d'amélioration. Merci de donner librement votre opinion; ce questionnaire sera traité de manière anonyme par l'Unité Pédagogique (http://www. unil.ch/fbm-up). Veuillez cocher, pour chaque question, la case qui correspond à votre opinion et ajouter vos remarques complémentaires à la fin.

Important !
Ces formulaires étant saisis automatiquement, merci de les remplir de la façon suivante :

\begin{tabular}{|c|c|c|c|c|c|}
\hline & non & $\begin{array}{c}\text { plutôt } \\
\text { non }\end{array}$ & $\begin{array}{c}\text { plutôt } \\
\text { oui }\end{array}$ & oui & $\begin{array}{l}\text { sans } \\
\text { avis }\end{array}$ \\
\hline 1. Les objectifs du cours ont été présentés & 0 & 0 & 0 & 0 & O \\
\hline 2. Le cours était bien structuré & 0 & 0 & 0 & 0 & 0 \\
\hline 3. Le cours était bien adapté à vos connaissances préalables & O & O & O & O & O \\
\hline 4. La quantité d'informations présentées était adaptée au temps à disposition & ○ & O & O & O & O \\
\hline 5. Il y avait suffisamment d'illustrations (exemples, cas, expériences, etc.) & O & O & O & O & O \\
\hline 6. Les notions importantes ont été suffisamment développées & O & O & O & O & O \\
\hline 7. Votre réflexion a été stimulée & 0 & O & O & 0 & O \\
\hline $\begin{array}{l}\text { 8. L'utilisation des supports didactiques (slides, tableaux, films, etc.) vous a aidé.e à } \\
\text { mieux comprendre les notions enseignées }\end{array}$ & 0 & 0 & 0 & O & O \\
\hline 9. Le cours était présenté de façon intéressante & 0 & O & ○ & 0 & O \\
\hline $\begin{array}{l}\text { 10. Les explications de l'enseignant.e vous ont permis de comprendre les notions } \\
\text { présentées }\end{array}$ & 0 & 0 & 0 & 0 & 0 \\
\hline $\begin{array}{l}\text { 11. L'enseignant.e a accepté volontiers les questions ou l'expression d'opinion des } \\
\text { étudiant.e.s }\end{array}$ & O & 0 & O & 0 & O \\
\hline 12. Globalement, vous avez apprécié cet enseignement & 0 & 0 & 0 & 0 & 0 \\
\hline
\end{tabular}

Quels étaient, selon vous, les points forts de cet enseignement?

Figure 2. Questionnaire adressé aux apprenants de l'Université de Lausanne pour évaluer l'enseignement

\section{Les différentes fonctions d'évaluation dans une classe inversée}

L'évaluation vise divers buts. Les fonctions de l'évaluation qui suivent ne sont guère exhaustives, mais relèvent les fonctions les plus utilisées par les enseignant.e.s de FLE/S. Elles sont toutes utilisées dans une classe inversée à des moments variés.

3.1. Qu'est-ce que l'évaluation au service de l'apprentissage? Aider à l'apprentissage 
L'évaluation au service de l'apprentissage (ou évaluation formative/formatrice) : elle sert à éclairer les enseignant.e.s sur ce que les apprenant.e.s comprennent et leur permet de planifier et d'orienter l'enseignement tout en fournissant une rétroaction utile aux apprenant.e.s. Quelques exemples d'évaluation formative méritent d'être mentionnés : le journal de bord, le portfolio, des exercices, une étude de cas, le projet collaboratif, les tâches, etc.

En tentant de trouver une définition pertinente de l'évaluation formative, celle de Scallon nous a retenu l'attention. L'évaluation formative est « un processus d'évaluation continue ayant pour objectif d'assurer la progression des individus engagés dans une démarche d'apprentissage ou de formation, selon deux voies possibles : soit par des modifications de la situation ou du contexte pédagogique, soit en offrant à chaque individu l'aide dont il a besoin pour progresser, et, ce, dans chacun des cas, pour apporter, s'il y a lieu, des améliorations ou des correctifs appropriés $^{9} \gg$ (Scallon, 2014).

Être évalué pour mieux apprendre est l'un des fondements même de la classe inversée ! Dans l'évaluation formative - si jamais une note est attribuée - c'est à titre indicatif qu'elle l'est (et ne comptera pas pour la moyenne). Ce qui nous amène dans cette partie à nous pencher sur l'importance de la remédiation, à comprendre et à approfondir la dynamique de la rétroaction dans une évaluation qu'elle soit formative ou sommative.

L'évaluation formative est utilisée à différentes fins, elle permet, entre autres de poser un diagnostic, d'orienter la régulation des apprentissages et de planifier des activités de remédiation (une démarche précieuse pour la classe inversée).

\subsection{Qu'est-ce que l'évaluation de l'apprentissage? Construire des jugements}

\section{L'évaluation de l'apprentissage (ou évaluation sommative) : elle consiste à} recueillir les renseignements à la suite de l'évaluation permettant aux apprenant.e.s, aux enseignant.e.s et aux parents, ainsi qu'à la communauté éducative au sens large, d'être informés sur les résultats d'apprentissage atteints à un moment précis afin de souligner les réussites, planifier les interventions et continuer à favoriser la réussite ${ }^{10}$.

Puisque le système d'éducation accorde une importance aux évaluations sommatives, plusieurs enseignant.e.s se sentiraient obligé.e.s d'enseigner « pour l'examen » aux dépens des objectifs d'apprentissage ${ }^{11}$.

L'évaluation sommative ou certificative dans une classe inversée peut être individuelle ou de groupe, doit respecter la cohérence pédagogique entre le système d'évaluation, les objectifs, la méthode pédagogique et l'outil et informe de la

\footnotetext{
${ }^{9}$ Gérard Scallon - L'évaluation formative des apprentissages - Québec, Canada, 2014.

${ }^{10}$ Éducation et formation, Manitoba, [http://www.edu.gov.mb.ca/m12/eval/role.html]

${ }^{11}$ Bruno Hubert (2015). Ibid.
} 
progression des apprentissages et des acquisitions effectuées des apprenant.e.s à la fin d'une étape d'un cursus scolaire ou académique.

L'évaluation sommative permet ainsi de confirmer ce que l'élève a appris, d'élaborer un classement, de voir si l'élève possède les qualifications requises pour passer à la prochaine année scolaire, mais surtout, s'il possède les prérequis pour l'obtention d'un diplôme ${ }^{12}$.

\subsection{Qu'est-ce que l'évaluation en tant qu'apprentissage? Réguler son apprentissage}

\section{L'évaluation en tant qu'apprentissage (ou auto-évaluation, régulation,} rétroaction, remédiation) permet aux apprenant.e.s de prendre conscience de leurs méthodes d'apprentissage et d'en profiter pour ajuster et faire progresser leurs apprentissages en assumant une responsabilité accrue à son égard (confer infra pour plus de détails).

\subsubsection{Qu'est-ce que la rétroaction ? Progresser et aller de l'avant}

En faisant un remue-méninge auprès d'un groupe d'enseignant.e.s de FLE/S pour compléter la question qui suit « selon vous, la rétroaction dans une classe inversée est de pouvoir... », les éléments qui ressortent ressemblent à :

- « ...encadrer les travaux des apprenants ?»,

- $\quad$ «...correspondre avec les apprenants ?»,

- $\quad$ «...animer un forum de discussion?»,

- «...rencontrer en privé les apprenants ?»,

- «...s'informer sur ce qui ne fonctionne pas dans le cours ?»,

- «...suggérer des pistes d'amélioration?», etc.

Selon Lambert, Rossier et Daele, 2009, la rétroaction se définit comme une information que l'enseignant.e fournit à l'apprenant.e à propos de la réalisation des tâches d'apprentissage. Cette rétroaction peut être réalisée dans un contexte formel ou informel ainsi qu'à différents moments du processus d'apprentissage.

La rétroaction devient un soutien à l'apprentissage et permet à l'apprenant.e de progresser ou d'aller de l'avant (feedforward), lorsqu'elle 1) donne l'occasion à l'apprenant.e de prendre du recul par rapport à un travail et qu'elle vise à l'améliorer. 2) Aide à préparer une évaluation finale ou sommative. 3) Fournit des clés à l'étudiant.e pour progresser par la suite de façon plus autonome.

\footnotetext{
${ }^{12}$ Bruno Hubert (2015). L'évaluation : un élément crucial du processus d'apprentissage [http://rire.ctreq.qc.ca/2015/06/evaluation-apprentissage/] (consulté en date du 24 janvier 2018)
} 
Ainsi en encadrant les travaux de ses apprenant.e.s, la rétroaction pousse l'enseignant.e à faire un retour constructif sur des travaux écrits longs ou courts, comme une expression écrite, un résumé, une argumentation, une dissertation, un mémoire, une thèse, etc.).

Les caractéristiques d'une rétroaction efficace $^{13}$ : elle propose des pistes d'amélioration concrètes, informe sur les forces, les réussites, les acquis ainsi que sur les défis réalistes à relever, elle est formulée de façon constructive, précise et adaptée au niveau de langage de l'apprenant.e, elle est soumise dans un court délai suivant la tâche (10-15 jours pour un travail long) et elle est en lien avec les objectifs d'apprentissage et les critères d'évaluation.

La rétroaction peut prendre également la forme d'une correspondance avec les apprenant.e.s où l'enseignant ressort en premier les points forts du travail rendu, signale les incohérences (ce qui ne fonctionne pas dans le travail rendu) et propose des pistes d'amélioration.

La rétroaction peut se faire auprès d'un apprenant.e que l'enseignant rencontre en privé ou d'un groupe d'apprenant.e. (via le forum par exemple).

3.3.2. Qu'est-ce que la remédiation? Réguler d'une façon permanente son apprentissage

La remédiation est une activité de régulation (ou d'autorégulation) des apprentissages qui a pour objectif de pallier les lacunes et les difficultés relevées lors de la rétroaction.

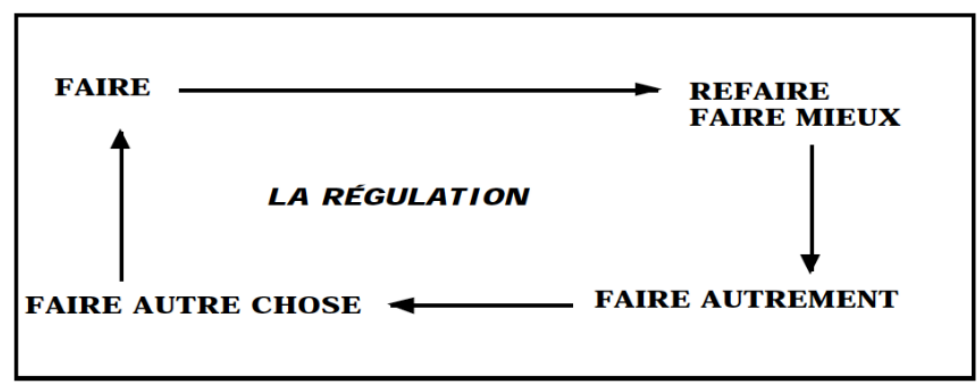

Figure 3. Michel Vial, Conceptions de la régulation et apprentissage, Les cahiers de 1977

\footnotetext{
${ }^{13}$ La Rétroaction : élément phare pour l'apprentissage de nos étudiants, Claude Boucher, UQAT, Le Tableau, Volume 4, numéro 3, 2015
} 
Quatre grandes catégories de remédiation sont identifiées par Jean-Marie De Ketele et Paquay (1991) allant des plus légères aux plus profondes.

1. Les remédiations par feedback/rétroaction précise, ciblée, détaillée ${ }^{14}$.

2. Les remédiations par répétition ou par travaux complémentaires (révision de la partie concernée ${ }^{15}$, remédiation par un travail complémentaire sur la partie concerné $e^{16}$, par révision des prérequis non maîtrisés ${ }^{17}$, remédiation reprenant ou consolidant les prérequis concernant la discipline ${ }^{18}$.

3. Les remédiations par adoption de nouvelles stratégies d'apprentissage (activités d'exploration reprenant certains apprentissages fondamentaux, activités d'apprentissage systématique pour entrainer l'apprenant à utiliser une règle, un procédé, une formule, une technique, activités d'intégration pour mobiliser des acquis en situation).

4. Les actions sur des facteurs plus fondamentaux comme des décisions d'ajustement prises en conseil de classe à propos des facteurs scolaires interférant avec les apprentissages ${ }^{19}$, des attitudes visant l'amélioration du climat éducationnel (absence de moyens didactiques, formation des enseignants, etc.) et du climat institutionnel (effectif de classes, conseil d'enseignement, de coordination, etc.) ou des décisions d'ajustement à propos de facteurs extrascolaires nécessitant le recours à des personnes extérieures ${ }^{20}$.

\section{L'évaluation des apprentissages et la rétroaction : un moment d'apprentissage dans une évaluation formative}

Comme susmentionné, la rétroaction peut trouver sa place à la fois dans l'évaluation formative et sommative. La première (l'évaluation formative) apporte un accompagnement et un encouragement à l'apprenant, de l'information sur ses acquis/ses compétences en construction et permet de situer sa progression/son évolution par rapport à un objectif donné. La deuxième (évaluation sommative)

${ }^{14}$ De Ketele et Paquay évoquent les exemples suivants pour un feedback: Communiquer à l'apprenant la correction (remplacer «en couleur " par « en colère ", «L'un frappe l'autre et l'autre frappe l'un» par "ils se disputent». La remédiation peut prendre la forme d'une autocorrection : donner à l'apprenant les outils pour s'autocorriger (des critères, un référent tel un URL vers une encyclopédie. La remédiation peut avoir la forme aussi la forme d'une confrontation entre une autoévaluation et le corrigé donné par l'enseignant ou d'autres apprenants. Par exemple, l'enseignant peut proposer à l'apprenant un canevas d'une lettre administrative pour l'amener à donner une meilleure organisation typographique à son texte ou des ensembles lexicaux pour l'améliorer et l'enrichir.

${ }^{15}$ Réviser par exemple les adjectifs possessifs à la $1^{\text {ìre }}$ personne du singulier pour remédier aux erreurs comme « deux camarades des moi », « la maison de moi ».

${ }^{16}$ D'autres exercices d'extension du fait de la langue, par exemple : les autres personnes de la conjugaison, varier la forme de l'adjectif possessif selon le possesseur et les objets possédés.

${ }^{17}$ Reprendre un apprentissage antérieur ainsi que les parties de l'apprentissage qui n'ont pas pu être bénéfiques vu la non maitrise initiale de ces prérequis, exemple reprise de l'étude des verbes pronominaux (à sens réciproque) : se battre, se disputer, se parler, se frapper, se quereller, s'écrire, etc.

${ }^{18}$ Exemple : distinguer entre une lettre personnelle et une lettre administrative (formules

d'adresse, de politesse), la ponctuation, le temps de la narration, etc.

${ }^{19}$ Par exemple des capacités cognitives de base nécessitant des réapprentissages fondamentaux et des décisions de réorientation (apprenant accusant un retard en matière de lecture et d'écriture).

${ }^{20}$ Exemple les parents pour un suivi scolaire, les psychologues pour le trouble du comportement, les orthophonistes pour les problèmes de dyslexie, de dysgraphie, les thérapeutes pour les maladies chroniques, etc.

XLinguae, Volume 14 Issue 4, October 2021, ISSN 1337-8384, eISSN 2453-711 85 
regroupe des énoncés liés à l'évaluation des apprentissages ${ }^{21}$ (travaux, examens, tests, exposés ou autres formes de contrôles) ainsi que des énoncés portant sur la rétroaction faite, suite aux évaluations.

Trois moments forts de la rétroaction seront développés dans la partie qui suit : l'évaluation diagnostique (1.1.), la régulation immédiate (1.2), et enfin la gestion des courriels et des rencontres (1.3). Ces moments ne sont pas exhaustifs dans une évaluation formative, et sont choisis à titre indicatif.

\subsection{L'évaluation diagnostique ${ }^{22}$ dans une classe inversée}

\subsubsection{Qu'est-ce que l'évaluation diagnostique?}

Dans le tableau ci-dessous se trouve la définition de l'évaluation diagnostique (quoi ?), les raisons pour lesquelles elle est utilisée (pourquoi ?), le déroulement des tâches (comment ?), à quel moment se pratique-t-elle (quand ?) ainsi que des exemples d'activités utilisées en évaluation diagnostique (activités?).

\begin{tabular}{|l|l|l|l|l|}
\hline Quoi? & Pourquoi ? & Comment? & Quand? & Activités ? \\
\hline $\begin{array}{l}\text { Évaluer les acquis } \\
\text { préalables des } \\
\text { apprenants : ce } \\
\text { qu'ils savent déjà } \\
\text { est-il mis en place, } \\
\text { leurs compétences, } \\
\text { etc. }\end{array}$ & $\begin{array}{l}\text { Mieux } \\
\text { connaître ses } \\
\text { apprenants } \\
\text { (fournir un état } \\
\text { des lieux sur } \\
\text { leurs } \\
\text { compétences } \\
\text { et } \\
\text { connaissances) }\end{array}$ & $\begin{array}{l}\text { Tâches } \\
\text { correspondant } \\
\text { aux objectifs du } \\
\text { cours mais pour } \\
\text { lesquelles ils } \\
\text { sont peu } \\
\text { préparés }\end{array}$ & $\begin{array}{l}\text { Au début d'un } \\
\text { trimestre, } \\
\text { d'une année, } \\
\text { d'un chapitre } \\
\text { ou d'une leçon }\end{array}$ & $\begin{array}{l}\text { Quiz papier } \\
\text { (QCM) } \\
\text { Quiz en ligne } \\
\text { (plateforme } \\
\text { pédagogique) }\end{array}$ \\
\hline $\begin{array}{l}\text { Détecter/dépister } \\
\text { les représentations } \\
\text { impropres: erreurs } \\
\text { classiques et } \\
\text { pratiques } \\
\text { inappropriées à } \\
\text { remplacer }\end{array}$ & $\begin{array}{l}\text { Ancrer les } \\
\text { nouveaux } \\
\text { apprentissages } \\
\text { surles anciens }\end{array}$ & $\begin{array}{l}\text { Tâches } \\
\text { graduées et } \\
\text { révélatrices }\end{array}$ & & \\
\hline & $\begin{array}{l}\text { Remédier } \\
\text { rapidement } \\
\text { aux difficultés } \\
\text { rencontrées }\end{array}$ & $\begin{array}{l}\text { Évaluation ne } \\
\text { contribuant àla } \\
\text { note ! }\end{array}$ & & $\begin{array}{l}\text { Mises en } \\
\text { situation, } \\
\text { Exercices } \\
\text { sémantiques, } \\
\text { etc. }\end{array}$ \\
& & & & \\
& & & $\begin{array}{l}\text { Penser aussi } \\
\text { aux activités } \\
\text { aidant à } \\
\text { remédier au } \\
\text { besoin! } \\
\text { Etc. }\end{array}$ \\
\hline
\end{tabular}

Tableau 2. L'évaluation diagnostique

L'évaluation diagnostique n'a pas pour mission d'attribuer une note, mais de relever le niveau d'acquisition de l'apprenant.e sur des items, des connaissances ou

${ }^{21}$ Il convient de distinguer l'évaluation des apprentissages (les rendus des apprenants) de l'évaluation des enseignements/ou évaluation de l'enseignant (qui porte sur le cours et la façon avec laquelle il a été donné).

${ }^{22}$ Elle est aussi appelée évaluation prédictive. 
des compétences. Elle est utilisée dans une classe inversée soit à domicile après visionnement d'une capsule vidéo, ou précédent un texte à lire, et constitue, comme la figure ci-dessous le montre, un test préalable au service des apprenant.e.s et de l'enseignant.e. à la fois afin de rectifier chacun à sa façon le tir.

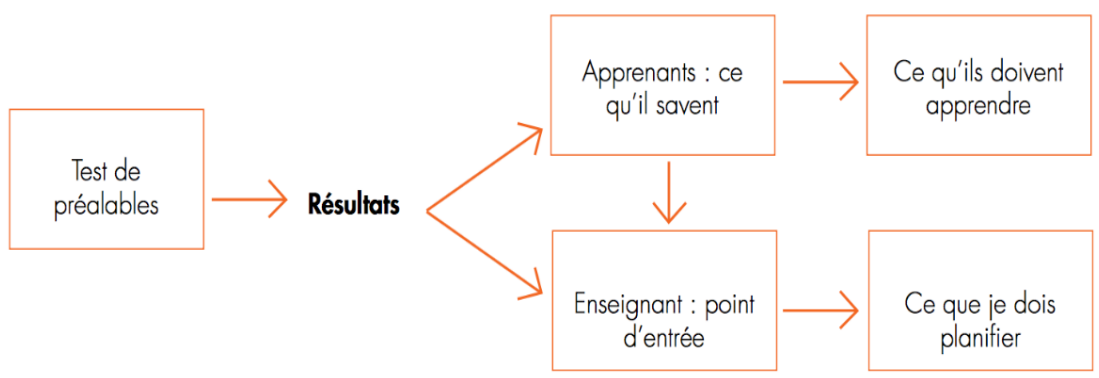

Figure 4. Évaluation des apprentissages scolaires, Développement de capacités pour l'éducation pour tous, UNESCO, Bamako, juin 2015, p.26

\subsubsection{Exemples d'évaluation diagnostique}

Trois exemples d'évaluation diagnostique seront examinés, à savoir les QCM ou questions à choix multiples, le point le plus nébuleux et l'exercice sémantique.

\section{Les Questions à Choix Multiples (QCM) (5 minutes)}

Les QCM nécessitent la compréhension des concepts enseignés. Les apprenant.e.s écrivent leurs réponses, puis travaillent en dyades ${ }^{23}$ pour en discuter. Les QCM sont utiles pour évaluer la compréhension des apprenant.e.s, déterminée par le nombre de bonnes réponses. Les QCM permettent aussi à l'enseignant.e de mieux structurer son cours.

Le point le plus nébuleux (vague, obscur, confus) (1-2 minutes)

L'enseignant.e demande aux apprenant.e.s de nommer sur un petit papier le point le moins clair du cours et sur lequel ils souhaiteraient revenir au début de la séance prochaine.

Un exercice sémantique (5-10 minutes)

Dans un cours de français de l'entreprise par exemple, l'enseignant.e peut demander aux apprenant.e.s de noter individuellement ce qu'est et ce que n'est pas pour eux une entreprise, et ensuite de discuter en binôme ce qui manque aux critères trouvés, et enfin d'essayer d'en donner une définition.

${ }^{23}$ En groupe de deux, en binôme

XLinguae, Volume 14 Issue 4, October 2021, ISSN 1337-8384, eISSN 2453-711 7 


\begin{tabular}{|l|l|}
\hline Ce qu'est l'entreprise pour moi & $\begin{array}{l}\text { Pour moi, ce que n'est pas } \\
\text { l'entreprise ... }\end{array}$ \\
\hline & \\
\hline & \\
\hline & \\
\hline
\end{tabular}

Figure 5. Exercice sémantique

\subsection{La régulation immédiate en classe inversée}

\subsubsection{Qu'est-ce que la régulation immédiate?}

Dans le tableau ci-dessous se trouvent les éléments principaux d'une définition de la régulation immédiate (quoi ?), les raisons pour lesquelles elle est utilisée (pourquoi ?), le déroulement des tâches (comment ?), à quel moment (quand ?) ainsi que des exemples d'activités utilisées en régulation immédiate (activités ?).

\begin{tabular}{|c|c|c|c|c|}
\hline Quoi? & Pourquoi? & Comment? & Quand? & Activités? \\
\hline \multirow[t]{3}{*}{$\begin{array}{l}\text { Échanger } \\
\text { constamment de } \\
\text { façon structurée } \\
\text { ou non avec les } \\
\text { apprenants }\end{array}$} & $\begin{array}{l}\text { Contrôler la } \\
\text { progression } \\
\text { des } \\
\text { apprentissages }\end{array}$ & $\begin{array}{l}\text { Observer } \\
\text { les } \\
\text { apprenants }\end{array}$ & Tout le temps! & $\begin{array}{l}\text { Questions / } \\
\text { réponses } \\
\text { (Q/R), } \\
\text { Points de } \\
\text { discussion } \\
\text { dansles } \\
\text { exposés, } \\
\text { Télévoteurs } 24\end{array}$ \\
\hline & $\begin{array}{l}\text { Contrôler la } \\
\text { réalisation des } \\
\text { tâches } \\
\text { prescrites }\end{array}$ & $\begin{array}{l}\text { Questionner } \\
\text { les } \\
\text { apprenants }\end{array}$ & $\begin{array}{l}\text { Durant les } \\
\text { exposés, durant } \\
\text { les travaux } \\
\text { individuels ou } \\
\text { d'équipe en } \\
\text { classe, à la fin } \\
\text { d'une classe, au } \\
\text { début d'une } \\
\text { classe (sur le } \\
\text { travail réalisé } \\
\text { entre les cours), } \\
\text { ou même entre } \\
\text { les cours! }\end{array}$ & $\begin{array}{l}\text { Exiger et } \\
\text { commenter } \\
\text { des plans de } \\
\text { travail }\end{array}$ \\
\hline & $\begin{array}{l}\text { Apporter une } \\
\text { remédiation } \\
\text { immédiate. }\end{array}$ & $\begin{array}{l}\text { Obtenir des } \\
\text { traces du } \\
\text { travail } \\
\text { (processus } \\
\text { et produit) } \\
\text { des } \\
\text { étudiants }\end{array}$ & & $\begin{array}{l}\text { Faire créer } \\
\text { des schémas } \\
\text { (carte } \\
\text { conceptuelle) } \\
\text { Forums de } \\
\text { discussion, } \\
\text { Papier } \\
\text { minute } \\
\text { Etc. }\end{array}$ \\
\hline
\end{tabular}


Tableau 3. La régulation immédiate

\subsubsection{Exemples de régulation immédiate}

Trois exemples de régulation immédiate seront considérés ci-dessous à savoir le papier minute appelé aussi le questionnaire minute, le rappel libre périodique et la minute de réflexion écrite.

\section{Le questionnaire minute (1 minute)}

À la fin de la séance du cours, l'enseignant demande aux apprenant.e.s de répondre à deux questions :

- Qu'est-ce que vous avez appris de plus important dans le cours aujourd'hui $? »$

- et Quelle est la question à laquelle vous n'avez pas obtenu de réponse?

L'enseignant.e. lit les réponses afin de donner une rétroaction et de répondre aux questions pendant le cours suivant.

Le rappel libre périodique (3 minutes)

L'enseignant.e. demande aux apprenants de compléter les trois phrases suivantes

- $\quad$ Le point le plus important est...

- Un bon exemple de l'application de ce point est...

- Ce que je ne saisis pas très bien est...

L'enseignant.e alloue deux ou trois minutes, puis demande aux apprenants de faire part de leurs points.

La minute de réflexion écrite (5 minutes)

(Pense, compare avec ton binome, partage avec le groupe ${ }^{24}$ )

L'enseignant.e pose une question à laquelle les apprenant.e.s répondent individuellement par écrit. Ils/elles partagent ensuite les réponses en dyades, et enfin, ils.elles partagent leurs réflexions avec la classe.

\subsection{Les courriels et rencontres en classe inversée}

Dans le tableau ci-dessous se trouve une explication de comment les courriels et rencontres entre apprenant.e et enseignant.e peuvent contribuer à faire de la rétroaction un moment d'apprentissage dans une évaluation formative. Ainsi sera observé le (quoi ?), les raisons pour lesquelles elle est utilisée (pourquoi ?), le déroulement des tâches (comment ?), à quel moment se pratique-t-elle (quand ?) ainsi que des exemples d'activités utilisées lors des rencontres ou à travers les courriels (activités ?).

\footnotetext{
${ }^{24}$ En anglais, l'activité s'appelle "Think-Write-Pair \& Share".
} 


\begin{tabular}{|c|c|c|c|c|}
\hline Quoi? & Pourquoi? & Comment? & Quand? & Activités? \\
\hline \multirow[t]{3}{*}{$\begin{array}{l}\text { Répondre aux } \\
\text { questions d'un } \\
\text { apprenant, faire } \\
\text { face à ses } \\
\text { difficultés } \\
\text { d'apprentissage ou } \\
\text { autres }\end{array}$} & $\begin{array}{l}\text { Diagnostiquer, } \\
\text { Encourager, } \\
\text { clarifier, } \\
\text { Echanger }\end{array}$ & $\begin{array}{l}\text { Courriels? } \\
\text { Déterminez à } \\
\text { l'avance: } \\
\text { fonction de ces } \\
\text { échanges et } \\
\text { délai de vos } \\
\text { réponses. }\end{array}$ & $\begin{array}{l}\mathrm{A} \\
\text { l'initiative } \\
\text { de } \\
\text { l'apprenant, } \\
\text { généraleme } \\
\text { nt }\end{array}$ & $\begin{array}{l}\text { Beaucoup } \\
\text { de } \\
\text { courriels? }\end{array}$ \\
\hline & $\begin{array}{l}\text { Encadrer, } \\
\text { Réévaluer, } \\
\text { etc. }\end{array}$ & $\begin{array}{l}\text { Rencontre(s)? } \\
\text { Au-delà du } \\
\text { factuel, } \\
\text { favoriser une } \\
\text { saine } \\
\text { motivation } \\
\text { (contrôlabilité) }\end{array}$ & & $\begin{array}{l}\text { Foire aux } \\
\text { questions } \\
\text { (FAQ) sur } \\
\text { la } \\
\text { plateforme } \\
\text { pédagogiqu } \\
\text { e }\end{array}$ \\
\hline & $\begin{array}{l}\text { S'ajuster, } \\
\text { Annoter } \\
\text { Attention : ce } \\
\text { n'est pas une } \\
\text { alternative à } \\
\text { l'absence (de } \\
\text { présence, } \\
\text { d'effort) de } \\
\text { l'apprenant. }\end{array}$ & & & $\begin{array}{l}\text { Réponses } \\
\text { préparées }\end{array}$ \\
\hline
\end{tabular}

Tableau 4. Les courriels et rencontres en classe inversée

\section{5. Évaluation des enseignements et rétroaction dans l'évaluation formative}

Dans cette partie, il convient de mentionner l'importance de la rétroaction en mi session (ou en cours de trimestre) faite par les apprenant.e.s. L'enseignant.e recueille les éléments d'information durant un échange organisé avec ses apprenant.e.s dans le but d'améliorer sa prestation d'enseignement actuelle et celles à venir.

L'avis sur la rétroaction en cours de trimestre (Groupe sur l'évaluation de l'enseignement, 2005) a documenté que les enseignant.e.s obtiennent de meilleurs résultats d'évaluation en fin de trimestre lorsqu'ils/elles ont recours à une forme de rétroaction en cours de trimestre (Overall et Marsh, 1979, notamment) et que le processus utilisé gagne à être volontaire, réfléchi et souple.

\subsection{Questions de réflexion}

S'il fallait trouver 2 raisons pour obtenir de la rétroaction sur votre enseignement en cours de trimestre, quelles seraient-elles? (N’hésitez pas à les écrire)

$$
\begin{aligned}
& 1 . \\
& 2 .
\end{aligned}
$$

S'il fallait penser à 3 questions que vous vous posez par rapport à l'efficacité de votre enseignement, quelles seraient-elles? (N’hésitez pas à les écrire) 
2.

Comment vous y êtes-vous pris pour obtenir de la rétroaction de vos apprenants?

1.

2.

Comment avez-vous reçu la rétroaction donnée par vos apprenants?

1.

2.

Quel est l'intérêt d'obtenir de la rétroaction en cours?

1 .

2.

Comment avez-vous communiqué les résultats à vos apprenants?

1.

2.

\subsection{Qu'est-ce que la rétroaction en mi session?}

Dans le tableau ci-dessous, se trouvent les éléments principaux d'une définition de la rétroaction en mi session (quoi ?), les raisons pour lesquelles elle est utilisée (pourquoi ?), le déroulement des tâches (comment ?), à quel moment (quand ?) ainsi que des exemples d'activités utilisées en lors d'une rétroaction (activités ?).

\begin{tabular}{|l|l|l|l|l|}
\hline Quoi ? & Pourquoi ? & Comment ? & Quand ? & Activités ? \\
\hline $\begin{array}{l}\text { Mettre entre } \\
\text { courenthèse pour } \\
\text { échanger avec les } \\
\text { apprenants sur } \\
\text { celui-ci }\end{array}$ & $\begin{array}{l}\text { Détecter et } \\
\text { régler les } \\
\text { problèmes et les } \\
\text { difficultés } \\
\text { pendant la durée } \\
\text { d'un cours }\end{array}$ & $\begin{array}{l}\text { Questionnaire } \\
\text { écrit ou en } \\
\text { ligne, ouvert } \\
\text { ou fermé, } \\
\text { anonyme et } \\
\text { volontaire } \\
\text { session ou } \\
\text { un peu avant } \\
\text { la mi session }\end{array}$ & $\begin{array}{l}\text { Questionnaire } \\
\text { ouvert en } \\
\text { quatre points } \\
\text { cardinaux }\end{array}$ \\
\hline & $\begin{array}{l}\text { Améliorer le } \\
\text { climat du cours } \\
\text { et l'évaluation } \\
\text { de fin de } \\
\text { trimestre }\end{array}$ & $\begin{array}{l}\text { Discussion } \\
\text { animée par un } \\
\text { tiers ou soi- } \\
\text { même }\end{array}$ & $\begin{array}{l}\text { Questionnaire } \\
\text { fermé mais } \\
\text { anonyme }\end{array}$ \\
\hline & $\begin{array}{l}\text { Faire réfléchir } \\
\text { les apprenants } \\
\text { sur leur } \\
\text { apprentissage. }\end{array}$ & & & \\
\hline
\end{tabular}

Tableau 5. La rétroaction en mi session

XLinguae, Volume 14 Issue 4, October 2021, ISSN 1337-8384, eISSN 2453-71181 
Il convient de donner deux exemples l'un portant sur le questionnaire ouvert en quatre points cardinaux et l'autre qui met en valeur le questionnaire semi guidé de rétroaction de mi session.

\section{Exemple de questionnaire à quatre points cardinaux}

Cette fiche vise à améliorer le cours et votre apprentissage. Merci de donner librement votre opinion.

\begin{tabular}{|l|l|}
\hline \multicolumn{1}{|c|}{ Points positifs } & \multicolumn{1}{|c|}{ Points à améliorer } \\
\hline Enseignant et cours & Enseignant et cours \\
\hline Apprenant(e) & Apprenant (e) \\
\hline
\end{tabular}

Figure 6. Fiche de rétroaction points cardinaux

\section{$\underline{\text { Exemple de questionnaire semi guidé de rétroaction de mi session }}$}

Dans le cadre du cours Français Langue des affaires «FRS1150 » où nous avons intégré la classe inversée, dans le but d'améliorer votre expérience éducative et de répondre de la meilleure façon à vos attentes et à vos besoins, je voudrais avoir votre rétroaction sur des questions reliées au cours.

Cette évaluation de l'efficacité de l'enseignement est anonyme et volontaire. Je vous encourage à donner votre point de vue sur le déroulement du cours et sur les moyens d'en faire une expérience encore plus significative pour votre apprentissage. Soyez sûrs que vos réponses seront prises en considération et qu'elles seront traitées objectivement et scientifiquement. Je vous ferai part des résultats lors de notre prochaine rencontre.

Soyez le plus sincère possible ; votre feed-back constructif est important : veuillez remplir le tableau suivant, puis donnez votre opinion sur les trois points essentiels identifiés dans la seconde partie. Vous pouvez ajouter d'autres commentaires dans la troisième partie.

Ce cours, répond-il à vos attentes ? Votre opinion est très importante !

\begin{tabular}{|l|l|l|}
\hline $\begin{array}{l}\text { À commencer / à } \\
\text { mettre en place }\end{array}$ & $\begin{array}{l}\text { À interrompre / à } \\
\text { abandonner }\end{array}$ & À poursuivre \\
\hline & & \\
& & \\
& & \\
\hline
\end{tabular}


De manière générale :

Le cours répond à mes attentes ?
$\begin{aligned} & \text { L'enseignante explique bien la } \\ & \text { matière? }\end{aligned}$
$\begin{aligned} & \text { L'ambiance - environnement de } \\ & \text { classe est positif et favorise } \\ & \text { l'apprentissage ? }\end{aligned}$

Autres remarques :

Merci de votre coopération et de votre rétroaction!

5.3. Que dit la littérature sur la rétroaction en mi session ${ }^{25}$ ? suivants :

Les top 6 réponses dans la littérature de la rétraction en mi session sont les

1. [L'enseignant.e peut] obtenir des renseignements importants sur le rendement des [apprenants] ainsi que la façon de percevoir le cours (Yao \& Grady, 2006)

2. [La rétroaction peut] servir de motivation pour les apprenants, leur permettre de se sentir contributeurs au sein d'un cours (Hampton \& Reiser, 2002)

3. [La rétroaction peut] informer sur les ajustements que vous et vos apprenants pourriez faire pour améliorer l'expérience d'apprentissage/enseignement (Hampton \& Reiser, 2002)

4. [La rétroaction peut] renforcer la relation professeur-étudiant (Angelo \& Cross, 1993)

5. [La rétroaction peut] offrir un canal de communication alternatif (par écrit) aux apprenants qui seraient, autrement, trop timides pour partager (Angelo \& Cross, 1993)

6. [La rétroaction peut] conduire généralement à la hausse des évaluations de fin de semestre (Davies, 1993)

Ci-dessous deux témoignages d'enseignant.e.s sur la rétroaction en mi session qui consolident la littérature susmentionnée

Enseignant 1

"Lors de la rétroaction en cours de session, je reçois souvent des commentaires sur la pédagogie du cours. Certains apprenants adorent déjà la formule de classe

\footnotetext{
${ }^{25}$ Appelée aussi « rétroaction d'étape" à des fins d'amélioration de l'enseignement en tant que rétroaction sur l'enseignement en cours de session.
}

XLinguae, Volume 14 Issue 4, October 2021, ISSN 1337-8384, eISSN 2453-71183 
inversée qui les poussent à travailler, mais d'autres réclament davantage de cours magistraux. Ça me permet de m'arrêter et de leur expliquer pourquoi, ils n'auront pas de cours magistraux. Ce n'est guère pour les ennuyer, c'est parce que je pense que de m'écouter leur expliquer de manière claire et limpide comment on accorde le participe passé, ils vont sortir de mon cours avec l'impression que c'est facile à accorder le participe passé. Mais quand ils vont être confrontés à ce genre de problème devant leur feuille, ils vont avoir de la difficulté. Alors c'est ce que j'appelle le syndrome du prof trop clair. Une fois que l'on a expliqué quelque chose très clairement, les étudiants n'ont plus à travailler pour la réussir. C'est pour cela que je tiens à ce que l'aspect plus théorique, magistral, explicatif soit fait avant et qu'en classe, on utilise nos connaissances... La rétroaction de mi session est un moment privilégié pour mettre les points sur les $i$ et répondre ou pas aux attentes des apprenants en leur expliquant certainement le pourquoi ».

\section{Enseignant 2}

"La rétroaction de mi session est l'occasion de rectifier le tir avant que ce ne soit trop tard. Quand je reçois la rétroaction finale sur le cours (donc l'évaluation de l'enseignement), j'ai beaucoup moins de commentaires parce que les étudiants ont compris l'intérêt de l'activité de rétroaction et ont vu que la plupart de leurs attentes ou demandes a été prise en considération. En revanche, je pense que tous les cours et tous les contenus ne se prêtent pas à cette approche-là non plus. Donc il faut avoir choisi un contenu qui s'y prête bien et avoir utiliser les avantages à leur meilleur. "

Après avoir vu l'évaluation des apprentissages, il convient d'étudier l'évaluation des apprentissages et l'importance de la rétroaction dans une démarche sommative et plus spécifiquement les annotations écrites de textes.

\section{6. Évaluation des apprentissages et rétroaction dans l'évaluation sommative}

Dans le tableau ci-dessous, se trouvent les éléments principaux d'une définition des annotations écrites de textes (quoi ?), les raisons pour lesquelles elles sont utilisées (pourquoi ?), le déroulement (comment ?), à quel moment (quand ?) ainsi que des exemples d'activités d'annotation (activités ?). 


\begin{tabular}{|l|l|l|l|l|}
\hline Quoi? & Pourquoi ? & Comment ? & Quand? & Activités ? \\
\hline $\begin{array}{l}\text { Annoter des } \\
\text { travaux et des } \\
\text { examens, en } \\
\text { plus } \\
\text { d'attribuer } \\
\text { une note (le } \\
\text { cas échéant) }\end{array}$ & $\begin{array}{l}\text { Autelà de } \\
\text { justifier la note, } \\
\text { l'annotation } \\
\text { informe } \\
\text { l'apprenant de } \\
\text { ses forces et } \\
\text { faiblesses, }\end{array}$ & $\begin{array}{l}\text { Référer à grilles, } \\
\text { Référer à des } \\
\text { barèmes liés aux } \\
\text { objectifs du } \\
\text { cours, } \\
\text { Utiliser un code } \\
\text { explicite. }\end{array}$ & $\begin{array}{l}\text { Au moment } \\
\text { de la } \\
\text { correction, au } \\
\text { cas où le } \\
\text { travail ou } \\
\text { l'examen sera } \\
\text { transmis aux } \\
\text { apprenants. }\end{array}$ & $\begin{array}{l}\text { Annotations des } \\
\text { copies } \\
\text { électroniques } \\
\text { Annotation avec } \\
\text { logiciels dédiés } \\
\text { Donner un } \\
\text { corrigé type }\end{array}$ \\
& $\begin{array}{l}\text { Identifier des } \\
\text { correctifs }\end{array}$ & $\begin{array}{l}\text { Renvoyer à des } \\
\text { références du } \\
\text { cours ou du } \\
\text { domaine. }\end{array}$ & $\begin{array}{l}\text { Exemple de } \\
\text { code de } \\
\text { correction } \\
\text { (FLE) }\end{array}$ \\
\hline & $\begin{array}{l}\text { Supporter la } \\
\text { motivation et } \\
\text { augmenter la } \\
\text { satisfaction }\end{array}$ & $\begin{array}{l}\text { Éviter les termes } \\
\text { vagues ou } \\
\text { émotifs, } \\
\text { équilibrer les } \\
\text { commentaires. }\end{array}$ & & \\
\hline
\end{tabular}

Tableau 6. Les annotations écrites de textes

\section{La différenciation en évaluation ${ }^{26}$ : condition essentielle à la lutte contre l'échec dans une classe inversée}

7.1. Qu'est-ce que la différenciation pédagogique?

Le terme de pédagogie différenciée veut désigner « un effort de diversification méthodologique susceptible de répondre à la diversité des élèves. » (Louis LEGRAND, La différenciation pédagogique, Scarabée, CEMEA, Paris 1984). Pour Philippe Meirieu, « Différencier, c'est avoir le souci de la personne sans renoncer à celui de la collectivité. »(P. MEIRIEU, Enseigner, scenario pour un métier nouveau, ESF, 1989)

Selon le MELS ${ }^{27}$, dans L'évaluation des apprentissages au secondaire cadre de référence, la différenciation pédagogique «est avant tout une manière de penser l'enseignement, l'apprentissage et l'évaluation, une philosophie qui guide l'ensemble des pratiques pédagogiques. C'est une façon d'exploiter les différences et d'en tirer avantage ${ }^{28}$.

\footnotetext{
${ }^{26}$ MELS, L'évaluation des apprentissages au secondaire cadre de référence, Québec, Canada, 2006, p. 27.

${ }^{27}$ MELS : ministère de l'Éducation, du Loisir et du Sport

${ }^{28} \mathrm{Ibid}, \mathrm{p} .27$
}

XLinguae, Volume 14 Issue 4, October 2021, ISSN 1337-8384, eISSN 2453-71185 
Pour sa part, le Conseil supérieur de l'éducation indique que la différenciation pédagogique " est une démarche qui consiste à mettre en œuvre un ensemble diversifié de moyens et de procédures d'enseignement et d'apprentissage afin de permettre à des élèves d'âges, d'aptitudes, de compétences et de savoir-faire hétérogènes, d'atteindre par des voies différentes des objectifs communs et, ultérieurement, la réussite éducative ${ }^{29} »$.

Les enseignant.e.s ont depuis toujours constaté les différences entre les apprenant.e.s de leur classe et tenté de répondre le mieux possible aux besoins de chacun. Chaque enseignant.e doit en effet, à l'intérieur de sa pratique quotidienne, faire face à la difficulté d'accompagner des jeunes différents les uns des autres. Dans leur pratique, les enseignant.e.s recourent à une différenciation souvent intuitive et spontanée. Le défi actuel est donc de rendre cette différenciation plus formelle et surtout planifiée, soutenue par une intention pédagogique claire et concertée.

\subsection{Quelques principes de différenciation pédagogique en évaluation au sein d'une classe inversée}

La différenciation profite à tous les élèves, qu'ils soient particulièrement doués ou en grande difficulté. Comme la figure ci-dessous le montre, une flexibilité pédagogique s'impose. Sans changer la nature ni les exigences de ce qui est évalué, un aménagement et des modifications pour les apprenant.e.s ayant des besoins particuliers.

L'outil peut être varié pour atteindre les mêmes objectifs, chacun à son rythme. Ci-dessous se trouvent quelques exemples d'outils (un projet, une étude de cas courte/longue, une résolution de problèmes, une dissertation, des rapports de lecture, un article, des QCM/Réponse courte, des Questions à développement, une simulation, un jeu de rôle, un exposé oral, un débat, un portfolio, des bilans réflexifs, etc.

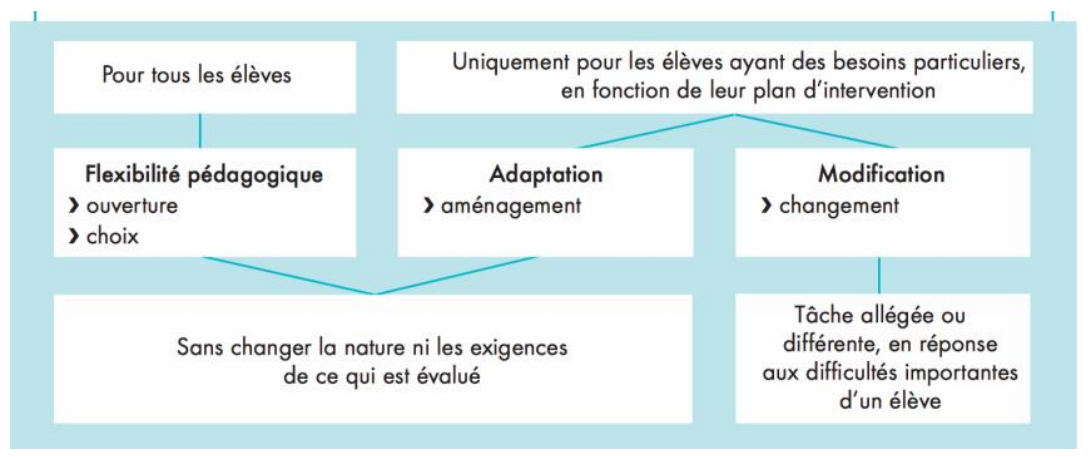

Figure 7. L'évaluation des apprentissages au secondaire cadre de référence, MELS, Québec, Canada, 2006, p. 29.

\footnotetext{
${ }^{29}$ Ibid., p. 27.
} 
La question qui se pose est comment différencier. On sait qu'un individu apprend mieux si un défi raisonnable lui est proposé. Si le défi est trop simple, il se démotive et s'il est trop élevé, il décroche. Il convient donc pour de viser le juste milieu pour l'apprenant et de l'amener à utiliser sa pleine potentialité.

Le Schéma ci-dessous traitant de la différenciation dans un apprentissage inclusif, dans une conception universelle de l'apprentissage, met en valeur trois domaines de différenciation à savoir 1) l'enseignant.e peut offrir plusieurs moyens de représentations (sur le plan de la perception, sur le plan de la langue et sur le plan de la compréhension). 2) L'enseignant.e peut offrir plusieurs moyens d'action et d'expression (sur le plan de l'action physique, sur le plan de la communication et sur le plan des fonctions exécutives). 3) L'enseignant.e peut offrir plusieurs moyens d'engagement pour éveiller l'intérêt, pour soutenir l'effort et la persévérance et sur le plan de l'autorégulation.

\begin{tabular}{|c|c|c|}
\hline $\begin{array}{l}\text { I. Offrir Plusieurs Moyens de } \\
\text { Représentation }\end{array}$ & $\begin{array}{l}\text { II. Offrir Plusieurs Moyens } \\
\text { D'Action et D'Expression }\end{array}$ & $\begin{array}{l}\text { III. Offrir Plusieurs Moyens } \\
\text { D'Engagement }\end{array}$ \\
\hline $\begin{array}{l}\text { 1: Offrir diverses possibilités sur le plan de la } \\
\text { perception } \\
\text { 1.1 Proposer divers moyens de personnaliser la } \\
\text { presentation de l'information } \\
\text { 1.2 Proposer d'autres modes de présentation pour les } \\
\text { informations auditives } \\
\text { 1.3 Proposer d'autres modes de présentation pour les } \\
\text { informations visuelles }\end{array}$ & $\begin{array}{l}\text { 4: Offrir diverses possibilitiés sur le plan de l'action } \\
\text { physique } \\
\text { 4.1 Varier les méthodes de réaction et d'interaction } \\
\text { 4.2Optimiser l'accès aux outilis et aux technologies } \\
\text { de soutien }\end{array}$ & $\begin{array}{l}\text { 7: Offrir diverses possibilités pour éveiller l'intérét } \\
7.1 \text { Optimiser les choix individuels et l'autonomie } \\
7.2 \text { Optimiser la pertinence, la valeur pédagogique et } \\
\text { lauthenticté } \\
7.3 \text { Minimiser les risques et les distractions }\end{array}$ \\
\hline $\begin{array}{l}\text { 2: Offtrir diverses possibilités sur les plans de la langue, } \\
\text { des expressions mathématiques et des symboles } \\
2.1 \text { Clarifier le vocabulaire et les symboles } \\
2.2 \text { Clarifier la syntaxe et la structure } \\
2.3 \text { Soutenir le décodage des textes, de la notation } \\
\text { mathématique et des symboles } \\
2.4 \text { Faciliter la compréhension lors du passage d'une } \\
\text { langue à l'autre } \\
2.5 \text { Illustrer linformation et les notions àl'aide de } \\
\text { plusieurs supports }\end{array}$ & $\begin{array}{l}\text { 5: Offfir diverses possibilitiés sur les plans de } \\
\text { l'expression et de la communication } \\
5.1 \text { Utiliser plusieurs supports de communication } \\
5.2 \text { Utiliser plusieurs outilis d'élaboration et de } \\
\text { composition } \\
\text { 5.3 Développer les compétences gracee à un soutien } \\
\text { échelonné en situation de pratique et de } \\
\text { performance }\end{array}$ & $\begin{array}{l}\text { 8: Offrir diverses possibilités pour soutenir l'effort et } \\
\text { la perséverance } \\
\text { 8.1 Souligner limportance des buts et des objectiffs } \\
\text { 8.2 Varier les exigences et les ressources pour rendre } \\
\text { les défis plus stimulants } \\
\text { 8.3 Favoriser la collaboration et la communauté } \\
\text { 8.4 Augmenter le retour d'information pour une plus } \\
\text { grande maatrise }\end{array}$ \\
\hline $\begin{array}{l}\text { 3: Offrir diverses possibilités sur le plan de la } \\
\text { compréhension } \\
\text { 3.1 Activer les connaissances antérieures ou fournir } \\
\text { les connaissances de base } \\
\text { 3.2. Faire ressortirl les modéles, les caractérístiques } \\
\text { essentielles, les idées principales et les relations } \\
\text { entre les notions } \\
\text { 3.3 Guider le traitement, la visualisation et la } \\
\text { manipulation de linformation } \\
\text { 3.4 Maximiser le transfert et la généralisation }\end{array}$ & $\begin{array}{l}\text { 6: Offfir diverses possibilités sur le plan des fonctions } \\
\text { exécutives } \\
\text { 6.1 Guider l'élève dans l'établissement d'objectifis } \\
\text { appropriés } \\
\text { 6.2 Soutenir la planification et l'élaboration de stratégies } \\
\text { 6.3 Faciliter la gestion de l'information et des ressources } \\
\text { 6.4 Améliorer la capacité de l'apprenant d'assurer le } \\
\text { suivi de ses progrees }\end{array}$ & $\begin{array}{l}\text { 9: Offrir diverses possibilités sur le plan de } \\
\text { l'autorégulation } \\
\text { 9.1 Promouvoir les attentes et les idées qui optimisent } \\
\text { la motivation } \\
\text { 9.2 Développer les stratégies d'autorégulation et la } \\
\text { faculté d'adaptation de lélève } \\
\text { 9.3 Développer la capacité d'auto-évaluation et de } \\
\text { réflexion }\end{array}$ \\
\hline $\begin{array}{l}\text { Des apprenants débrouillards, } \\
\text { bien informés et compétents }\end{array}$ & $\begin{array}{l}\text { Des apprenants centrés sur } \\
\text { des objectifs stratégiques }\end{array}$ & Des apprenants motivés et déterminés \\
\hline
\end{tabular}

Tableau 5. Lignes directrices de la conception universelle de l'apprentissage 


\subsection{Exemples de pratiques évaluatives de différenciation pédagogique ${ }^{30}$}

Le portfolio de l'apprenant.e suscite la différenciation pédagogique et des pratiques évaluatives en un sens que chacun des apprenant.e.s va à son propre rythme et personnalise son apprentissage comme bon lui semble. Les apprenant.e.s deviennent des praticiens réflexifs, capables de réfléchir de manière critique sur leur pratique professionnelle et sur leur façon d'apprendre » (Dochy, 2001).

En écriture : l'enseignan.et peut différencier les contenus ou le sujet de la tâche, il/elle pourra offrir aux élèves deux sujets différents qui permettent le développement de la même compétence. Il peut également demander aux apprenant.e.s de produire un texte médiatique pour différents destinataires, texte plus ou moins étoffé selon le rythme de l'apprenant.e.

Pour l'analyse d'un territoire type (par exemple, la métropole) sera abordée à partir de différentes grandes villes. Avec un.e élève ayant des besoins particuliers, une modification de la situation pourrait s'avérer nécessaire : une partie de la tâche à faire pourrait être enlevée ou le nombre de critères d'évaluation, réduit.

En lecture, par exemple, l'enseignant.e pourrait diminuer le nombre de critères d'évaluation pour un.e élève ayant des besoins particuliers.

Pour le projet personnel d'orientation par exemple, les élèves seront amené.e.s à partager leur réflexion en ayant la possibilité de le faire de façons variées (projection multimédia, vidéo, etc.). La durée de la tâche à réaliser pourra être différente selon l'élève, certain.e.s poussant plus loin leur réflexion. La présentation de projets en enseignement moral permettra à des élèves de produire une affiche, à d'autres, de créer un sketch ou une chanson. Il est donc possible de différencier à la fois la production et le processus. Donner des choix parmi différentes propositions de création en arts permettra aussi de différencier productions et processus ${ }^{31}$.

\section{Conclusion}

En guise de conclusion, l'évaluation est un processus de regard réflexif sur son propre apprentissage. L'évaluation est supérieure à la note et son essence même est avant tout un accompagnement progressif et humain de nos apprenat.e.s.

\section{Bibliographic references}

ALLAL, L. 2013. L'évaluation, un pont entre enseignement et apprentissage à l'Université. Dans M. Romainville, R. Gaasdoué et M. Vantourout (dir.), Évaluation et enseignement supérieur. Bruxelles : De Boeck.

ALBERTA Education framework. 2016. The Guiding Framework for the Design and Development of Kindergarten to Grade 12 Provincial Curriculum (Programs of Study) Available online: https://open.alberta.ca/publications/9781460130971.

${ }^{30}$ Les exemples sont puisés de L'évaluation des apprentissages au secondaire cadre de référence, MELS, Québec, Canada, 2006, p. 34.

${ }^{31}$ Ibid.pp.34 et 35 . 
ALBERTA, Alberta Education, Direction de l'éducation française. 2010. Aide à la différenciation pédagogique (version anglaise : Making a difference: meeting diverse learning needs with differentiated instruction).

BERGMANN, J. - AARON, S. 2012. Flip your Classroom, Reach every student in every class every day, Iste \& ASCD.

BISSONNETTE, S. - CLERMONT, G. Faire la classe à l'endroit ou à l'envers ? Available online: http://formation-profession.org/pages/article/20/1/173.

BITTEL, L. R. 1991. Right on Time. The complete guide for time-pressured managers, Business Audio Series, Mc Graw-Hill Inc. Cassette Audio.

BLOOMFIELD, A. - TAUZIN, B. 2001. Affaires à suivre. Paris : Hachette FLE.

Bogost-Georgia, I. 2013. The Condensed Classroom. Available online : The Condensed Classroom. Récupéré le 30 mars 2018.

BOMBARDIERI, C. 1996. L'entreprise. Hachette FLE.

Cadre Européen Commun de Référence pour les langues, CECRL. 2001. Available online: https://rm.coe.int/16802fc3a8.

CENTER for Applied Special Technology. 2011. Universal Design for Learning guidelines version 2.0. Wakefield, MA: Author. Traduit par Bergeron, Rousseau et Leclerc. pp. 91-92.

CLAS, A. - HORGUELIN, P. A. 1991. Le français, langue des affaires (3ème édition). Montréal : McGraw-Hill Éditeurs.

DANILO, M. - PENFORNIS, J.-L. 1993. Le français de la communication professionnelle. Paris : CLE international.

DANILO, M. - TAUZIN, B. 1991. Le français de l'entreprise. Paris : CLE international.

DOCHY, F. 2011. A New Assessment Era: Different Needs, New Challenges. Learning and Instruction, 10(1),11-20.

DOI : 10.1016/S0959-4752(00)00022-0

EID, C. - ODDOU, M. - LIRIA, Ph. 2019. La classe inversée. Clé international, (en cours d'impression).

EID, C. 2016. Comment implanter des activités implicatives et des jeux sérieux en classe de FLE ? Donner âme aux grands groupes. Reflets AQEFLS, 33, 29-30.

GARDNER, H. 2009. Theory of Multiple Intelligences, Northern Illinois University, Faculty Development and Instructional Design Center, USA

KEME'ENUI et Simmons 1999, cités par Tremblay, S., Raymond, O. et Henderson, T., La Conception universelle de l'apprentissage en enseignement supérieur dans leur PowerPoint. Available online: https://www.usherbrooke.ca/performa/fileadmin/sites/performa/documents/40e/Atelie r_pedagogie.pdf.

LEGRAND, L. 1984. La différenciation pédagogique. Paris : Scarabée, CEMEA.

CADRE de référence. 2006. L'évaluation des apprentissages au secondaire, version préliminaire, Québec

JORRO, A.-CROCE-SPINELLI, H. 2010. Le développement de gestes professionnels en classe de français. Le cas de situations de lecture interprétative. In : Didactique du français, Pratiques linguistique, littérature, didactique, pp. 125-140.

MAYER, R. - CLARCK, R. 2011. Les 10 principes de l'apprentissage multimédia, University of California, Santa Barbara, USA.

MCQUARRIE, MC R. - STACK, C. 2003-2006. Differentiated Instruction: Provincial Research Review, Choice, complexity and creativity, Alberta Education, Canada.

MEIRIEU, P. 1989. Différencier la pédagogie, Introduction. In: Cahiers pédagogiques, 277. 
MELS, Ministère de l'Éducation, du Loisir et du Sport. 2006. L'évaluation des apprentissages au secondaire, Cadre de référence, version préliminaire, Québec, Canada.

NISSEN, E. 2006. Scénarios de communication en ligne dans des formations hybrides. In: Le Français dans le monde. Recherches et applications, vol. 40, pp. 4458.

POU, G. - SANCHEZ, M. 1993. Commerce/affaires, Entraînez-vous, niveau intermédiaire. Paris : CLE international.

TAUZIN, B. - DUBOIS, A.-L. 2006. Express, Le monde professionnel en Français. Chambre de Commerce et d'Industrie de Paris, Hachette FLE.

TREMBLAY, S. - RAYMOND, O. - HENDERSON, T. 2014-2015. La conception universelle de l'apprentissage (CUA) en enseignement supérieur, Guide à l'intention des nouveaux professeurs et chargé de cours, Projet inter ordre, Québec.

WAKEFIELD, M. A. 2011. Lignes directrices sur la conception universelle de l'apprentissage, (Universal Design for Learning - UDL) : texte intégral, Version 2.0, Universal Design for Learning Guidelines version,

YAICHE, F. 1996. Les Simulations globales. Paris : Mode d'emploi, Hachette.

Words: 6782

Characters: 46687 (25,94 standard pages)

Prof. Cynthia Eid

Présidente de la Fédération internationale de professeurs de français (FIPF)

Doyenne de l'École de formateurs, Groupe IGS, France

Professor - researcher, American Business School of Paris

University de Valenciennes

France

eidcynthia@gmail.com 